University of Nebraska - Lincoln

DigitalCommons@University of Nebraska - Lincoln

8-2002

\title{
Spin Injection into Amorphous Semiconductors
}

\author{
Evgeny Y. Tsymbal \\ University of Nebraska at Lincoln, tsymbal@unl.edu
}

V. M. Burlakov

Department of Materials, University of Oxford, Oxford, UK

Ivan I. Oleinik

University of South Florida, Tampa, Florida, oleynik@usf.edu

Follow this and additional works at: https://digitalcommons.unl.edu/physicstsymbal

Part of the Condensed Matter Physics Commons

Tsymbal, Evgeny Y.; Burlakov, V. M.; and Oleinik, Ivan I., "Spin Injection into Amorphous Semiconductors" (2002). Evgeny Tsymbal Publications. 10.

https://digitalcommons.unl.edu/physicstsymbal/10

This Article is brought to you for free and open access by the Research Papers in Physics and Astronomy at DigitalCommons@University of Nebraska - Lincoln. It has been accepted for inclusion in Evgeny Tsymbal Publications by an authorized administrator of DigitalCommons@University of Nebraska - Lincoln. 


\title{
Spin injection into amorphous semiconductors
}

\author{
E. Y. Tsymbal \\ Department of Physics and Astronomy, University of Nebraska-Lincoln, Lincoln, Nebraska 68588 \\ V. M. Burlakov \\ Department of Materials, University of Oxford, Parks Road, Oxford OX1 3PH, United Kingdom \\ I. I. Oleinik \\ Department of Physics, University of South Florida, Tampa, Florida 33620
}

(Received 10 May 2002; published 1 August 2002)

\begin{abstract}
Using a realistic model for the atomic and electronic structure of amorphous silicon, we explore spin injection into amorphous semiconductors. We calculate the spin-dependent conductance of magnetoresistive devices within the Landauer-Büttiker formalism including inelastic scattering. We find that reducing the density of injected carriers and increasing the spin polarization of the electrodes are favorable for spin injection, whereas inelastic scattering is detrimental, and show that the upper limit for magnetoresistance is given by Julliere's formula.

DOI: 10.1103/PhysRevB.66.073201

PACS number(s): 72.25.Hg, 61.43.Dq, 72.25.Dc, 73.40.Rw
\end{abstract}

The field of spin electronics, wherein manipulating spin degrees of freedom in magnetoresistive devices makes it possible to read and write nonvolatile information through magnetism, has aroused considerable interest. ${ }^{1}$ The discovery and successful exploitation of giant magnetoresistance (GMR) in metallic multilayers boosted this field (for a recent review on GMR see Ref. 2). Making use of semiconductors in spin electronics has the advantage of incorporating the magnetoresistive devices into existing semiconductor technologies. The feasibility of using semiconductors is supported by their capability to carry highly spin-polarized currents over long distances $^{3}$ and by the successful demonstration of electrical spin injection from magnetic dilute semiconductors. ${ }^{4,5} \mathrm{Re}$ cent discoveries of room-temperature spin injection from metallic Fe into $\mathrm{GaAs}^{6}$ and a large magnetoresistance in GaMnAs/AlAs tunnel junctions ${ }^{7}$ further stimulated an interest in this field.

A typical device for magnetoresistive applications consists of two ferromagnetic electrodes, which are used to inject and detect spin-polarized current in a semiconductor (e.g., Ref. 8). Injecting the spin-polarized current is due to a different electronic structure (e.g., the density of states at the Fermi energy) for up- and down-spin electrons in the ferromagnetic electrode. Detecting the spin-dependent current is achieved by changing the relative magnetization of the two electrodes.

Ferromagnetic electrodes based on dilute magnetic semiconductors, such as GaMnAs, have been successfully used for spin injection. ${ }^{4,5}$ An application of these materials in magnetoresistive devices is, however, limited due to low $\mathrm{Cu}$ rie temperature. ${ }^{9}$ A search for new semiconductors that remain ferromagnetic at room temperature is critical for the applications. As was predicted theoretically within the ballistic regime of conduction, epitaxially grown ferromagnet/ semiconductor/ferromagnet tunnel junctions with metallic ferromagnets can possess very high values of magnetoresistance. ${ }^{10}$ Unfortunately, this is not the case in the diffusive regime of conduction, which is more relevant to the experimental conditions and in which spin injection from a ferromagnetic metal into a semiconductor is suppressed by the resistivity mismatch between these materials. ${ }^{11}$ As was predicted recently, the latter problem may be solved by introducing a tunnel barrier at the ferromagnet/semiconductor interface $^{12,13}$ that seems to be supported by the experiments. ${ }^{6}$

In this paper, using a realistic microscopic model for a semiconductor-based magnetoresistive device, we calculate the magnetoresistance (MR) as a function of the electronic density of states (DOS) of the electrodes and inelastic scattering rate of carriers within the semiconductor. We predict the highest MR for low density and low scattering rates of injected carriers and demonstrate that the upper limit for magnetoresistance is described by Julliere's formula.

We develop an accurate model for the atomic and electronic structure of amorphous silicon $(a-\mathrm{Si})$, which serves as a representative semiconducting material for spin injection and ensures the diffusive regime of conduction that is relevant to experiments. In order to obtain the atomic structure of $a$-Si we simulate deposition of silicon atoms from a vapor phase using Metropolis Monte Carlo technique within a random network model. ${ }^{14}$ The structure is grown onto the (001) surface of crystalline $\mathrm{Si}(c-\mathrm{Si})$ substrate in a square box with the side of $6 a / \sqrt{2}=23.04 \AA$ in the [110] direction $(a$ $=5.43 \AA$ is the lattice parameter of $c-\mathrm{Si}$ in the diamond structure). Periodic boundary conditions are imposed in the $x-y$ plane parallel to the substrate. After depositing 1036 atoms we decouple the grown $a$-Si sample from the substrate, attach to the both sides of the sample two (001) monolayers of $c$-Si, and anneal the structure, keeping the attached $c$-Si monolayers crystalline during the annealing. The resulting $a$-Si sample has thickness of $46 \AA$ and the unit cell of 1180 $\mathrm{Si}$ atoms including the two monolayers of $c$-Si that serve for the coupling to the electrodes. The structural characteristics of the simulated $a$-Si sample, such as the average coordination number of 3.81-3.85, the shape of the radial distribution function, and the half-width of the bond angle distribution function of about $20^{\circ}$, are in excellent agreement with the experimental data. ${ }^{15}$ 


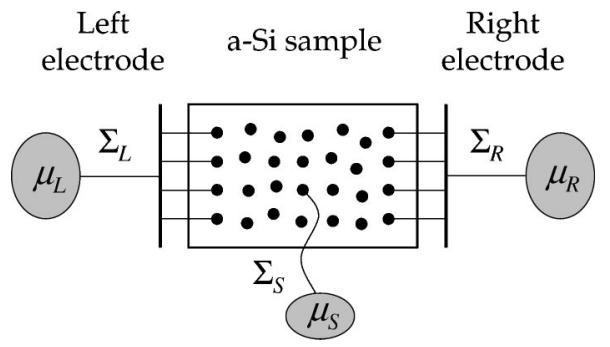

FIG. 1. Geometry of the system. The $a$-Si sample is attached to the left and right electrodes connected to reservoirs that are characterized by different chemical potentials $\mu_{L}$ and $\mu_{R}$. The electrodes affect the electronic structure of the $a$-Si sample through selfenergies $\Sigma_{L}$ and $\Sigma_{R}$. Inelastic scattering is included by connecting "scattering" electrodes to each atomic site and then by adjusting their chemical potentials $\mu_{S}$ to ensure current conservation throughout the sample.

We model the electronic structure of $a$-Si using a tightbinding approximation. The tight-binding Hamiltonian $H$ is constructed using the orthogonal $s p^{3}$ basis set. The bond integrals $\beta_{s s \sigma}, \beta_{s p \sigma}, \beta_{p p \sigma}$, and $\beta_{p p \pi}$, and the on-site atomic energies $E_{s}$ and $E_{p}$ for the $c$-Si structure are taken from Ref. 16. The bond integrals for the $a$-Si structure are scaled according to Ref. 17. This parametrization is designed to fit to various phases of $\mathrm{Si}$ and therefore accurately describes $a$-Si.

For calculating the conductance we use the LandauerBüttiker approach. ${ }^{18,19}$ We place the grown $a$-Si sample between two electrodes connected to reservoirs, as shown schematically in Fig. 1. The reservoirs are characterized by the equilibrium Fermi distributions with $\mu_{L}$ and $\mu_{R}$ being the chemical potentials of the left and right reservoirs, respectively. The electric current is driven in the sample by the applied voltage $V$, such that $\mu_{L}-\mu_{R}=e V$. Inelastic scattering is taken into account assuming that each atomic site of the $a$-Si sample is connected to a "scattering" electrode $S$ that serves as a phase-breaking scatterer, ${ }^{20,21}$ thereby introducing an incoherent component to the overall current flow (see Fig. 1). The current conservation throughout the sample is ensured by setting the chemical potentials $\mu_{S}$ of the scattering electrodes in a way that no net current flows into these electrodes.

Attaching the electrodes affects the electronic structure of the $a$-Si sample resulting in a broadening and a shift of the energy levels. This is taken into account by including selfenergy terms in the Green function

$$
G(E)=\left(E-H-\Sigma_{L}-\Sigma_{R}-\sum_{S} \Sigma_{S}\right)^{-1}
$$

where $\Sigma_{L}, \Sigma_{R}$, and $\Sigma_{S}$ are the self-energies associated with the left, right, and scattering electrodes and the summation is performed over all atoms of the sample. Imposing the requirement of no net current in the scattering electrodes and using the Landauer-Büttiker formalism within the linearresponse approximation, we obtain a system of linear equations for the chemical potentials:

$$
0=T_{L S}\left(\eta_{S}-1\right)+T_{R S} \eta_{S}+\sum_{S^{\prime}} T_{S S^{\prime}}\left(\eta_{S}-\eta_{S^{\prime}}\right) .
$$

Here $\eta_{S}=\left(\mu_{S}-\mu_{R}\right) / e V$ is the reduced chemical potential at site $S . T_{L S}, T_{R S}$, and $T_{S S^{\prime}}$ are the transmission coefficients, which can be found from ${ }^{19,21}$

$$
T_{\alpha \beta}=-\operatorname{Tr}\left[\left(\Sigma_{\alpha}-\Sigma_{\alpha}^{\dagger}\right) G\left(\Sigma_{\beta}-\Sigma_{\beta}^{\dagger}\right) G^{\dagger}\right]
$$

where indices $\alpha$ and $\beta$ denote $L, R, S$, or $S^{\prime}$. The net conductance per spin is given by

$$
\Gamma=\frac{e^{2}}{h}\left\{T_{L R}+\sum_{S}\left(1-\eta_{S}\right) T_{L S}\right\} .
$$

Within this approach all the properties of the ferromagnetic electrodes are contained in the self-energies $\Sigma_{L}$ and $\Sigma_{R}$, which are assumed to be spin dependent. In general, these self-energies are given by $\Sigma_{L, R}^{\uparrow, \downarrow}=V_{L, R} G_{L, R}^{\uparrow, \downarrow} V_{L, R}^{\dagger}$ for the up ( $\uparrow$ ) and down $(\downarrow)$ spins, where $G_{L, R}^{\uparrow, \downarrow}$ is the Green function of the left (right) electrode coupled to the sample by $V_{L, R}$. For simplicity we consider identical electrodes, for which $\Sigma^{\uparrow, \downarrow}=\Sigma_{R}^{\uparrow, \downarrow}=\Sigma_{L}^{\uparrow, \downarrow}$, and parametrize the self-energies, so that $\Sigma^{\uparrow, \downarrow}=-i \pi \beta_{s s \sigma}^{2} \rho^{\uparrow, \downarrow}$ are nonzero only for the $s$ orbitals (i.e., we assume that $G_{L, R}^{\uparrow, \downarrow}=-i \pi \rho^{\uparrow, \downarrow}$ and $\left.V_{L, R}=\beta_{s s \sigma}\right)$. Here $\rho^{\uparrow}$ and $\rho^{\downarrow}$ are the up-spin and down-spin DOS of the electrodes at the point of contact with the $a$-Si sample. The latter parametrization is similar to that used in Ref. 22. This formulation allows introducing a spin polarization of the electrodes, $P=\left(\rho^{\uparrow}-\rho^{\downarrow}\right) /\left(\rho^{\uparrow}+\rho^{\downarrow}\right)$, in the spirit of Julliere's model. $^{23}$

Figure 2(a) shows the calculated conductance (per cross section of the unit cell, $18 a^{2}$ ) as a function of electron energy. Here the energy interval corresponds to the band gap of bulk $c$-Si [a much broader energy range is shown in the inset in Fig. 2(a)]. We assume that the electrodes are ferromagnetic metals that are simulated by a relatively high density of states of $\rho=\rho^{\uparrow}+\rho^{\downarrow}=0.4 \mathrm{eV}^{-1} /$ atom and by the spin polarization $P=0.5$. As is seen from the thin solid line in Fig. 2(a), the conductance displays numerous resonances caused by localized states in the band gap. ${ }^{24}$ The thick line shows the effect of inelastic scattering introduced via parametrization of the self-energy of the scattering electrodes, so that $\Sigma_{S}=i \delta$. The parameter $\delta$ is set equal to $0.04 \mathrm{eV}$, which is a representative value for electron-phonon coupling in $a$-Si at room temperature (e.g., Ref. 25). As is evident from Fig. 2(a) that inelastic scattering smears out the resonant peaks enhancing the conductance on an average. The calculated value of the conductance per unit area at the Fermi energy $E_{F}$ $=0$, is about $4 \times 10^{8} \Omega^{-1} \mathrm{~cm}^{-1}$, which is in reasonable agreement with the experimental data for $a-\mathrm{Si}$ at room temperature. $^{25}$

Figure 2(b) shows the magnitude of MR versus electron energy for different DOS values of the electrodes in the presence of inelastic scattering, $\delta=0.04 \mathrm{eV}$. The MR is defined by the normalized difference between the conductance for the parallel and antiparallel magnetization of the electrodes, i.e., $\left(\Gamma_{P}-\Gamma_{\mathrm{AP}}\right) / \Gamma_{P}$. As can be seen from the inset in Fig. 2(b), for metallic electrodes with $\rho=0.4 \mathrm{eV}^{-1}$ the magnitude 


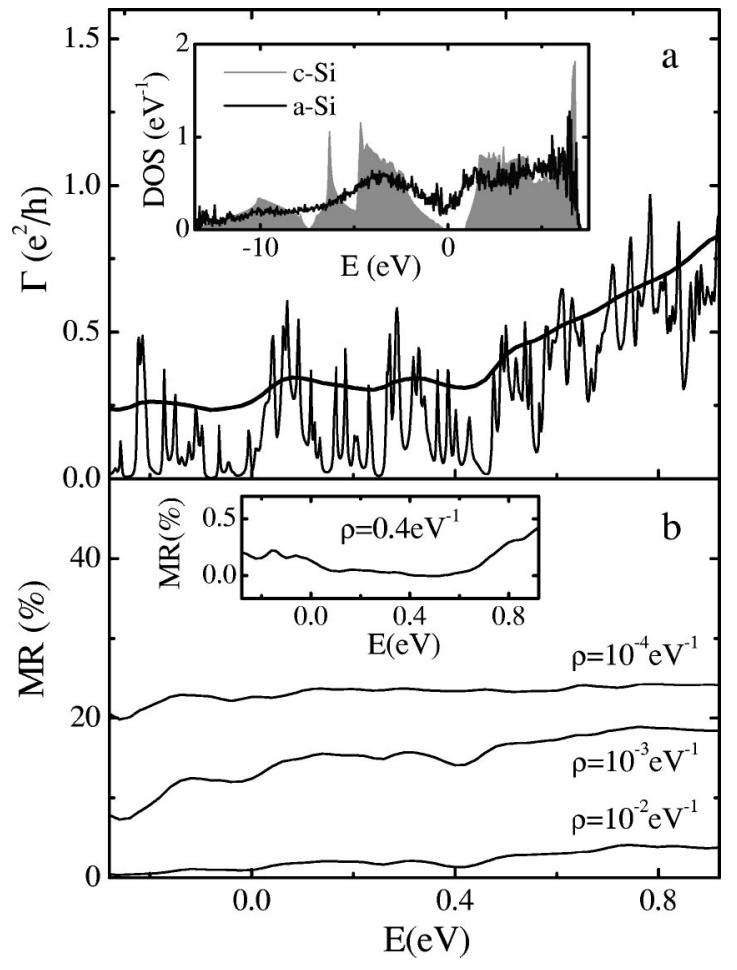

FIG. 2. Conductance (a) and magnetoresistance (b) versus electron energy for $P=0.5$. $\Gamma$ is plotted for $\delta=0$ (thin line) and $\delta$ $=0.04 \mathrm{eV}$ (thick line) for $\rho=0.4 \mathrm{eV}^{-1}$. The inset in (a) shows the DOS for $c$-Si and $a$-Si. MR is plotted for various values of $\rho$ and $\delta=0.04 \mathrm{eV}$. The Fermi energy lies at zero.

of MR is less than $0.5 \%$. The predicted low values of MR are in agreement with experimental observations and are typical for metallic electrodes (e.g., Ref. 26). In order to improve the magnetoresistive response of the device with metallic electrodes a very high spin polarization of the electrodes is necessary. This can be seen from Fig. 3, which shows the calculated MR as a function of the spin polarization $P$. For metallic electrodes $\left(\rho=0.1 \mathrm{eV}^{-1}\right.$ and $\rho=0.4 \mathrm{eV}^{-1}$ in Fig. 3) the MR becomes sizable only at a very high spin polarization, which is consistent with the prediction of Ref. 11.

The significant reduction of the DOS of the electrodes results in a dramatic enhancement of magnetoresistance. As is evident from Fig. 2(b), the MR increases gradually with decreasing $\rho$ for all the energies. For $P=0.5$ and $\rho$ $=10^{-4} \mathrm{eV}^{-1}$ it lies in the range of $20-25 \%$. Further reduction of the density of states does not change much the MR. The same tendency is evident from Fig. 3: with decreasing $\rho$ the MR increases for all the spin polarizations and eventually it saturates at values given by the curve for $\rho=10^{-5} \mathrm{eV}^{-1}$. The latter fact is illustrated in the inset of Fig. 3, which shows MR versus $\rho$ for $P=0.5$. As is seen, the highest values of MR can be achieved by reducing the DOS of the electrodes down to $\rho=10^{-5} \mathrm{eV}^{-1}$ or lower. We conclude, therefore, that the possibility of using magnetic semiconductor electrodes or tunneling contacts for the enhancement of spin injection $^{12,13}$ is the consequence of the low density of injected carriers.

The effect of the DOS of the electrodes on MR can be understood in terms of renormalization of the semiconductor energy levels due to the coupling to the electrodes. Indeed,

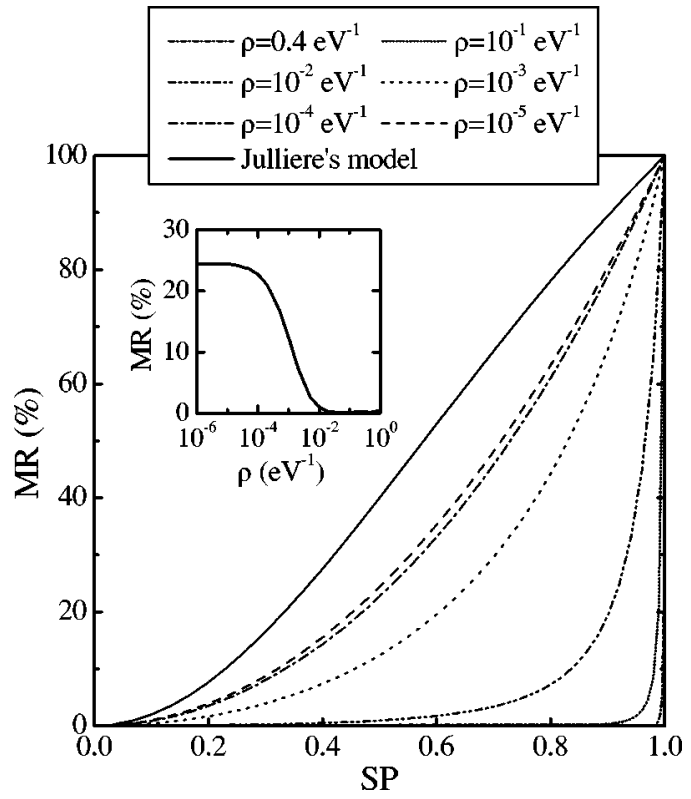

FIG. 3. Magnetoresistance as a function of the spin polarization of the electrodes for various values of $\rho$ and $\delta=0.04 \mathrm{eV}$. The solid line shows the result for Julliere's model that is indistinguishable from the result for $\rho=10^{-5} \mathrm{eV}^{-1}$ and $\delta=0$. The inset shows MR versus $\rho$ for $P=0.5$ and $\delta=0.04 \mathrm{eV}$.

for low DOS values the electrodes virtually do not disturb the energy spectrum of the semiconductor, which therefore acts as a linear transmitter with respect to the density of injected and detected carriers. In this regime the spin conductance is proportional to the product of the DOS for the left and right electrodes and the MR is given by Julliere's formula (the solid line in Fig. 3), which takes the form $2 P^{2} /\left(1+P^{2}\right)$ for identical electrodes. ${ }^{23}$ As our calculations show, in the absence of inelastic scattering the linear regime is achieved for $\rho \lesssim 10^{-5} \mathrm{eV}^{-1}$, thus indicating the range of applicability of Julliere's model. ${ }^{27}$ For higher DOS values the energy spectrum of the semiconductor is substantially affected by the electrodes making the injection and detection of spins highly nonlinear. This is due to the broadening of the semiconductor energy levels given by $\Sigma_{L}$ and $\Sigma_{R}$, which leads to the resonant mechanism of conduction. As was demonstrated in Ref. 24, the resonant conduction results in a decrease of magnetoresistance.

Similar to the DOS of the electrodes, an increase of inelastic scattering reduces MR. As is seen from Fig. 3, for $\delta$ $=0.04 \mathrm{eV}$ the MR is appreciably lower than for $\delta=0$ (compare the solid and dashed lines in Fig. 3 for $\rho=10^{-5} \mathrm{eV}^{-1}$ ). The mechanism, which is responsible for this decrease of $\mathrm{MR}$, originates from inelastic coupling between the localized states in the semiconductor. This coupling promotes conduction along quasi-one-dimensional chains of localized states, ${ }^{25}$ similar to that for multiresonance tunneling. ${ }^{24}$ We can conclude therefore that inelastic scattering is detrimental to MR, despite the fact that no spin-flip scattering is included in our model.

In conclusion, using a realistic microscopic model for amorphous silicon we have explored favorable conditions for spin injection into disordered semiconductors within the Landauer-Büttiker formalism. We found that magnetoresis- 
tance of spin-injection devices can be enhanced by decreasing the density of injected carriers, by increasing the spin polarization of the electrodes, and by reducing inelastic scattering within the semiconductor. The upper limit for magnetoresistance is given by Julliere's formula.
This research was supported by Hewlett-Packard Laboratories, Toppan Printing Co., and DARPA under Contract No. MDA972-00-1-0016. The computations were performed in the Materials Modelling Laboratory at the Department of Materials, University of Oxford.
${ }^{1}$ G. A. Prinz, J. Magn. Magn. Mater. 200, 57 (1999).

${ }^{2}$ E. Y. Tsymbal and D. G. Pettifor, in Solid State Physics, edited by H. Ehrenreich and F. Spaepen (Academic, New York, 2001), Vol. 56, pp. 113-237.

${ }^{3}$ J. M. Kikkawa and D. D. Awschalom, Nature (London) 397, 139 (1999).

${ }^{4}$ R. Fiederling, M. Keim, G. Reuscher, W. Ossau, G. Schmidt, A. Waag, and L. W. Molenkamp, Nature (London) 402, 787 (1999).

${ }^{5}$ Y. Ohno, D. K. Young, B. Beschoten, F. Matsukura, H. Ohno, and D. D. Awschalom, Nature (London) 402, 790 (1999).

${ }^{6}$ H. J. Zhu, M. Ramsteiner, H. Kostial, M. Wassermeier, H.-P. Schönherr, and K. H. Ploog, Phys. Rev. Lett. 87, 016601 (2001). ${ }^{7}$ M. Tanaka and Y. Higo, Phys. Rev. Lett. 87, 026602 (2001).

${ }^{8}$ S. Datta and B. Das, Appl. Phys. Lett. 56, 665 (1990).

${ }^{9}$ H. Ohno, J. Magn. Magn. Mater. 200, 110 (1999).

${ }^{10}$ J. M. MacLaren, X. G. Zhang, W. H. Butler, and X. Wang, Phys. Rev. B 59, 5470 (1999).

${ }^{11}$ G. Schmidt, D. Ferrand, L. W. Molenkamp, and A. T. Filip, B. J. van Wees, Phys. Rev. B 62, R4790 (2000).

${ }^{12}$ E. I. Rashba, Phys. Rev. B 62, R16 267 (2000).

${ }^{13}$ A. Fert and H. Jaffres, Phys. Rev. B 64, 184420 (2001).

${ }^{14}$ V. M. Burlakov, G. A. D. Briggs, A. P. Sutton, Y. Tsukahara, Phys. Rev. Lett. 86, 3052 (2001).

${ }^{15}$ K. Laaziri, S. Kycia, S. Roorda, M. Chicoine, J. L. Robertson, J. Wang, and S. C. Moss, Phys. Rev. Lett. 82, 3460 (1999); Phys. Rev. B 60, 13520 (1999).
${ }^{16}$ D. I. Chadi, J. Vac. Sci. Technol. 16, 1290 (1979).

${ }^{17}$ L. Goodwin, D. R. Skinner, and D. G. Pettifor, Europhys. Lett. 9, 701 (1989); D. R. Bowler, M. Fearn, C. M. Goringe, A. P. Horsfield, and D. G. Pettifor, J. Phys.: Condens. Matter 10, 3719 (1998).

${ }^{18}$ R. Landauer, IBM J. Res. Dev. 32, 306 (1988).

${ }^{19}$ M. Büttiker, IBM J. Res. Dev. 32, 317 (1988).

${ }^{20}$ M. Büttiker, Phys. Rev. B 33, 3020 (1986).

${ }^{21}$ S. Datta, Electronic Transport in Mesoscopic Systems (Cambridge University Press, Cambridge, 1995).

${ }^{22}$ H. Mehrez, J. Taylor, H. Guo, J. Wang, and C. Roland, Phys. Rev. Lett. 84, 2682 (2000).

${ }^{23}$ M. Julliere, Phys. Lett. 54A, 225 (1975).

${ }^{24}$ E. Y. Tsymbal and D. G. Pettifor, Phys. Rev. B 58, 432 (1998).

${ }^{25}$ Y. Xu, D. Ephron, and M. R. Beasley, Phys. Rev. B 52, 2843 (1995).

${ }^{26}$ P. R. Hammar, B. R. Bennett, M. J. Yang, and M. Johnson, Phys. Rev. Lett. 83, 203 (1999); S. Gardelis, C. G. Smith, C. H. W. Barnes, E. H. Linfield, and D. A. Ritchie, Phys. Rev. B 60, 7764 (1999).

${ }^{27}$ We note that our model can easily be generalized to the case of the orbital dependent $\rho$ and $\Sigma_{L, R}$. The upper limit for MR is, then, determined by Julliere's formula in which the orbitaldependent DOS is weighted with the corresponding bond integrals at the ferromagnet/semiconductor interface. 